Supporting Information

\title{
Impressive radiation stability of organic solar cells based on fullerene derivatives and carbazole-containing conjugated polymers
}

Ilya V. Martynov, ${ }^{\dagger}$ Alexander V. Akkuratov, ${ }^{\dagger}$ Sergey Yu. Luchkin, ${ }^{\S}$ Sergey A. Tsarev, ${ }^{\S}$ Sergei D.

Babenko,' Vladimir G. Petrov, ${ }^{\ddagger}$ Keith J. Stevenson ${ }^{\S}$ and Pavel A. Troshin $* \S \dagger$

† The Institute for Problems of Chemical Physics of the Russian Academy of Sciences, Semenov Prospect

1, Chernogolovka Moscow region, 142432, Russia.

$\S$ Skolkovo Institute of Science and Technology, Nobel St. 3, Moscow, 143026, Russia.

" The Institute for Energy Problems of Chemical Physics, Russian Academy of Sciences (Branch),

Semenov Prospect 1/10, Chernogolovka, Moscow region, 142432, Russia

${ }^{\ddagger}$ Lomonosov Moscow State University,GSP-1, Leninskie Gory, Moscow, 119991, Russian Federation.

*Corresponding Author

Pavel A. Troshin e-mail: P.Troshin@skoltech.ru 


\section{Table of Contents:}

Figure S1. GPC profiles of the P1 (a), P2 (b) and [60]PCBM (c) before and after exposure to gamma rays with the absorbed radiation dose of $200 \mathrm{~Gy}$.

Figurev S2. ESR spectra of the P1 (a), P2 (b) and [60]PCBM (c) before and after exposure to gamma rays with the absorbed radiation dose of $200 \mathrm{~Gy}$.

Figure S3-5. AFM topography, surface potential, phase and current mapping images of P1, P2 and [60]PCBM films before and after exposure to gamma rays.

Figure S6 EQE spectra of organic solar cells based on the blends of non-exposed S6 and exposed to 200Gy materials: P1/[60]PCBM and P2/[60]PCBM.

Figure S7 Evolution of the absorption and PL spectra of the films of P1/[60]PCBM, $\mathrm{P} 2+[60] \mathrm{PCBM}$.

Figures S8-9: AFM topography, surface potential, phase and current mapping images of P1/[60]PCBM and P2/[60]PCBM films before and after exposure to gamma rays

Figure S10. Voc vs. light intensity dependences for organic solar cells based on the pristine and exposed to gamma rays P1/[60]PCBM and P2/[60]PCBM blend films

Figure S11. Transient photovoltage (TPV) profiles for organic solar cells based on the pristine and exposed to gamma rays $\mathbf{P 1} /[60] \mathrm{PCBM}$ and $\mathbf{P 2} /[60] \mathrm{PCBM}$ blend films

Figure S12. Normalized characteristics of the solar cells based on P1+[60]PCBM and $\mathbf{P 1 + [ 7 0 ] P C B M}$ plotted as functions of the radiation dose.

Table S1 Characteristics of organic solar cells before and after exposure of the samples to different doses of gamma rays 
a)

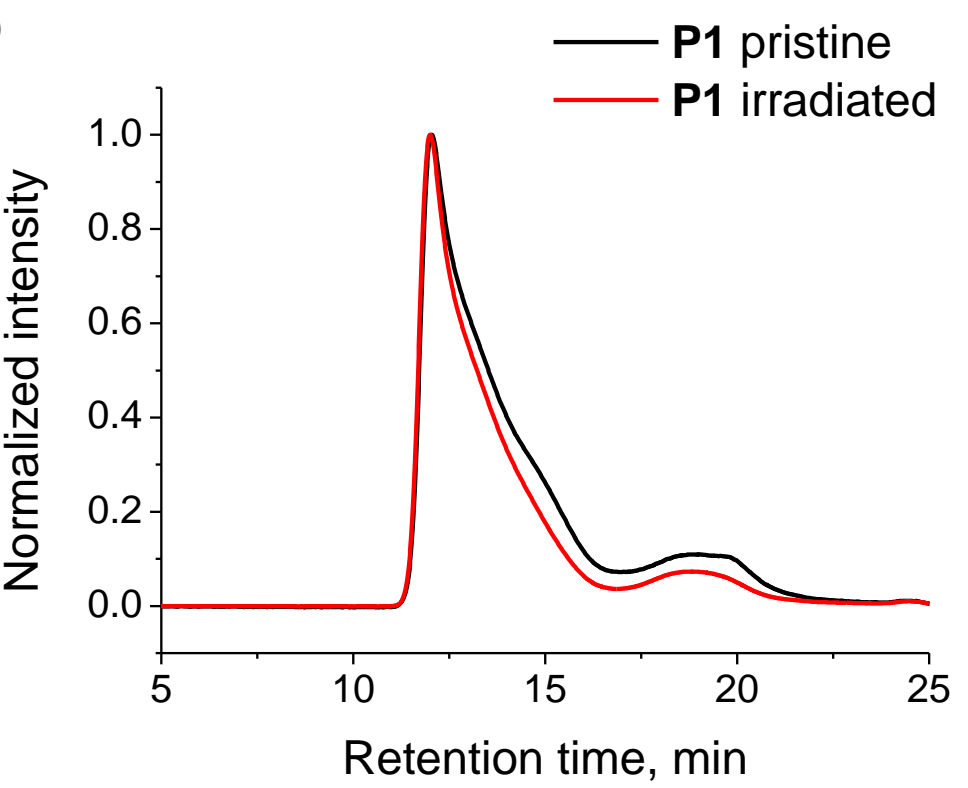

b)

P2 pristine

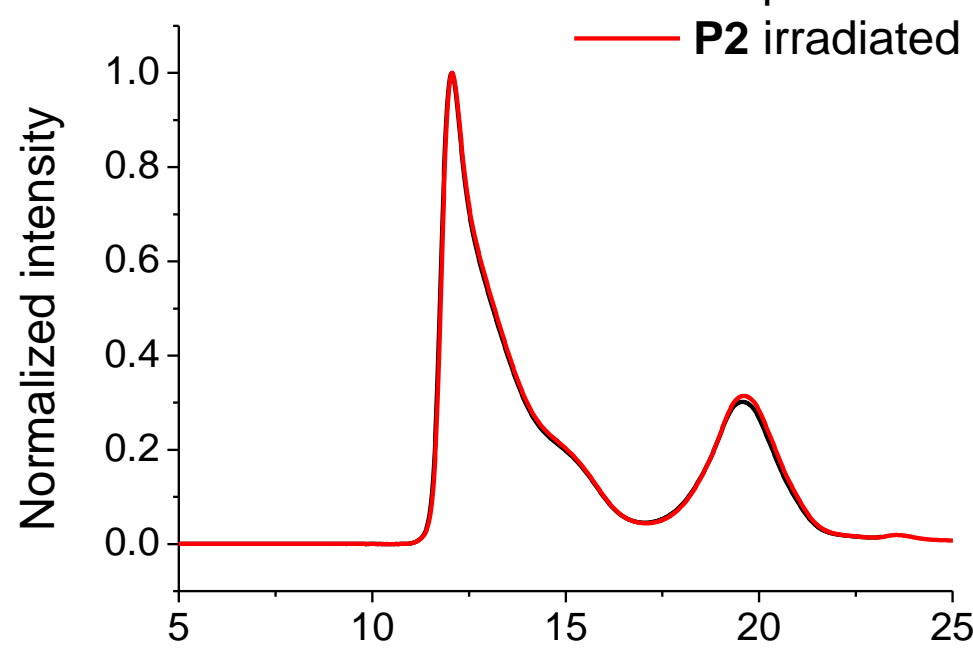

c)

Retention time, $\min$

[60]PCBM pristine

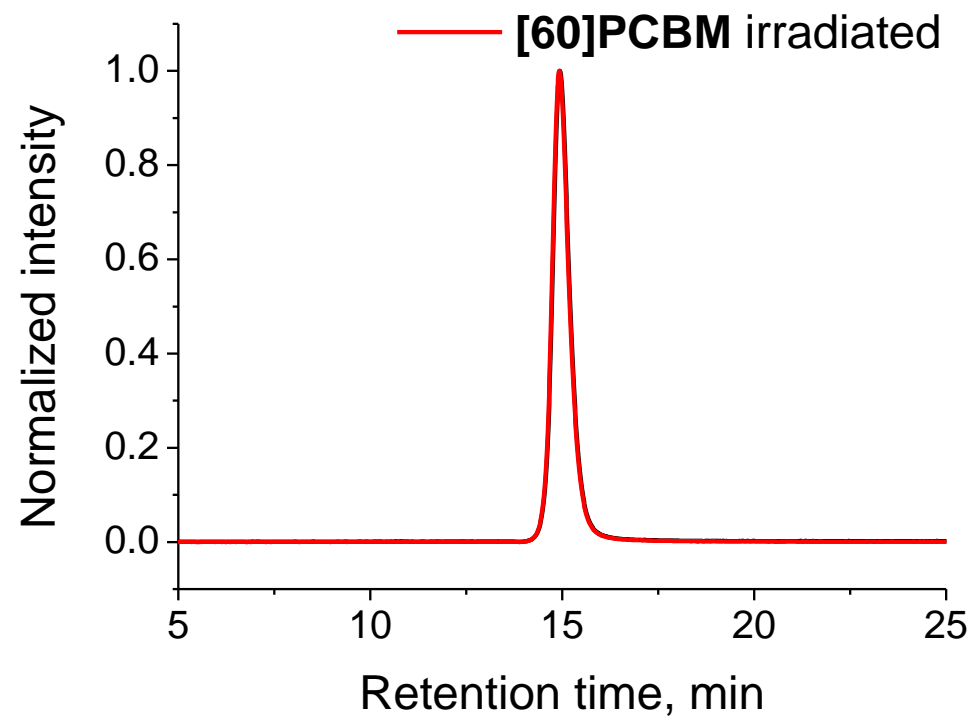

Figure S1. GPC profiles of the P1 (a), P2 (b) and [60]PCBM (c) before and after exposure to gamma rays with the absorbed radiation dose of $200 \mathrm{~Gy}$. 
a)

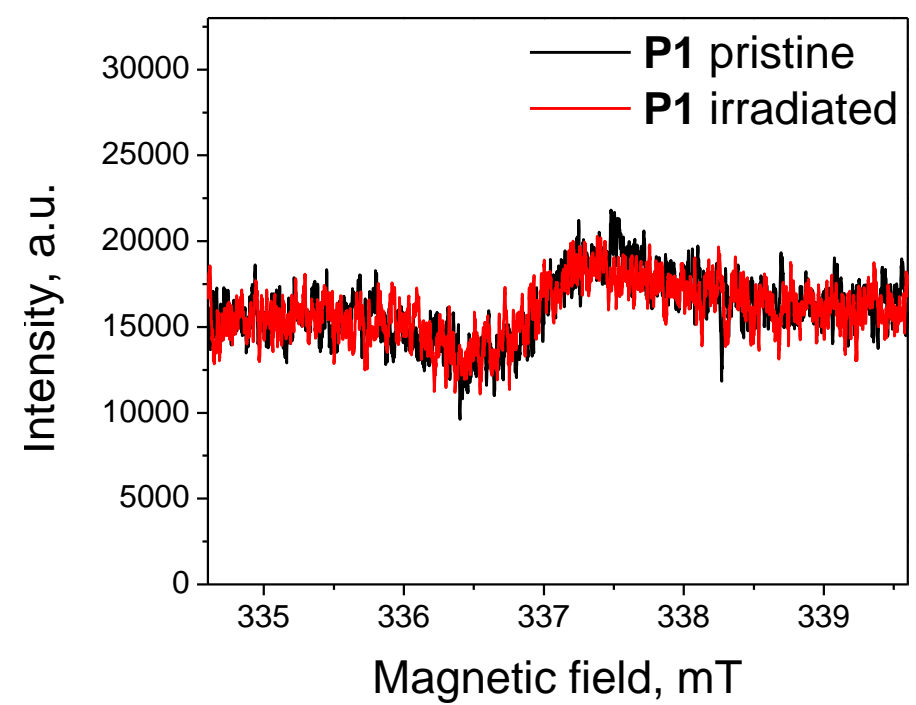

b)

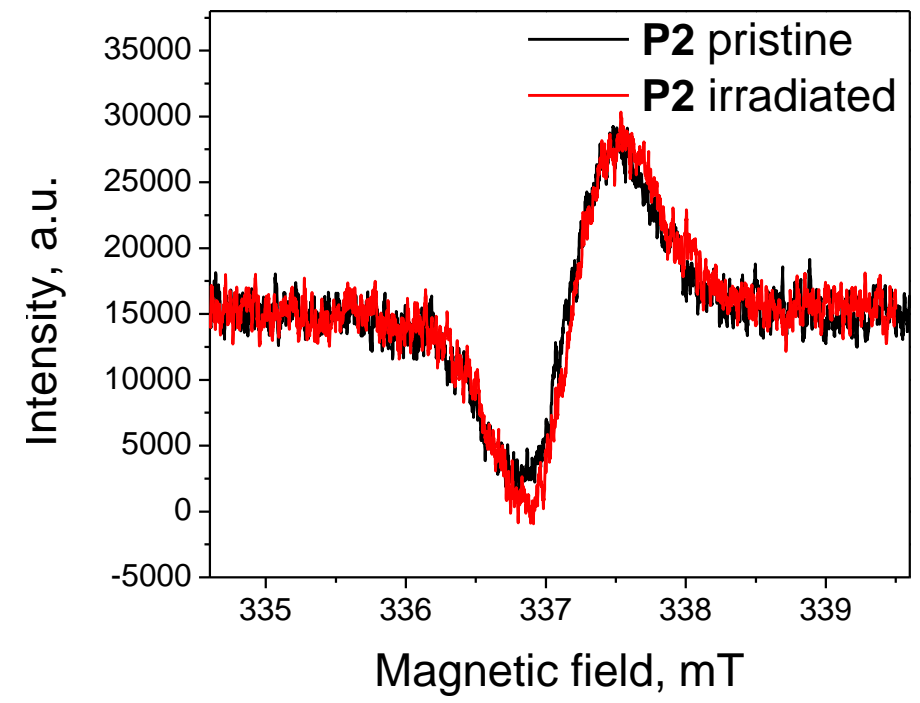

c)

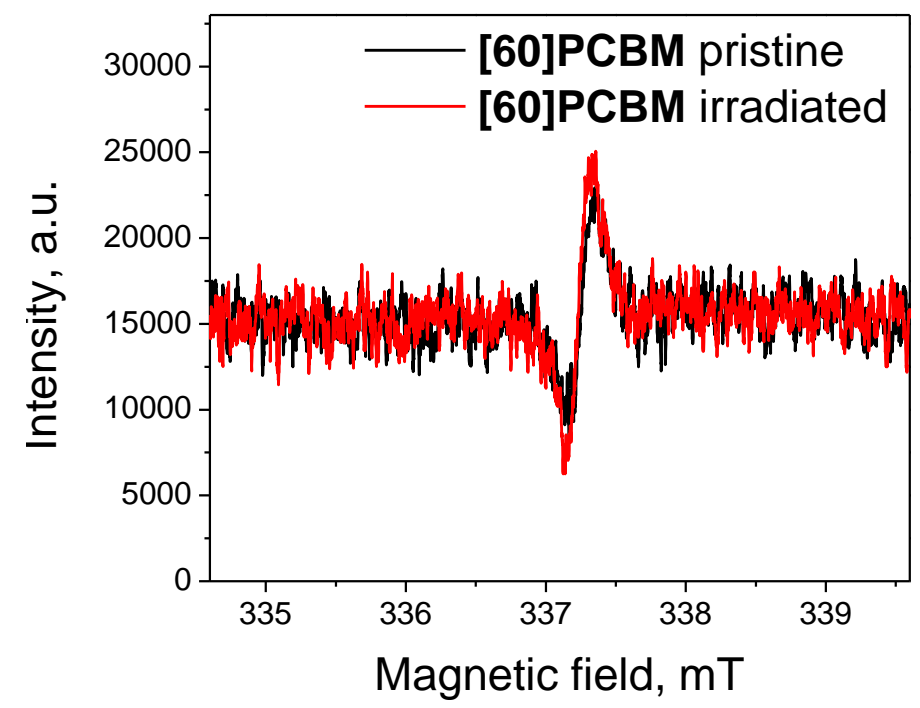

Figure S2. ESR spectra of the P1 (a), P2 (b) and [60]PCBM (c) before and after exposure to gamma rays with the absorbed radiation dose of $200 \mathrm{~Gy}$. 


\section{Pristine films}
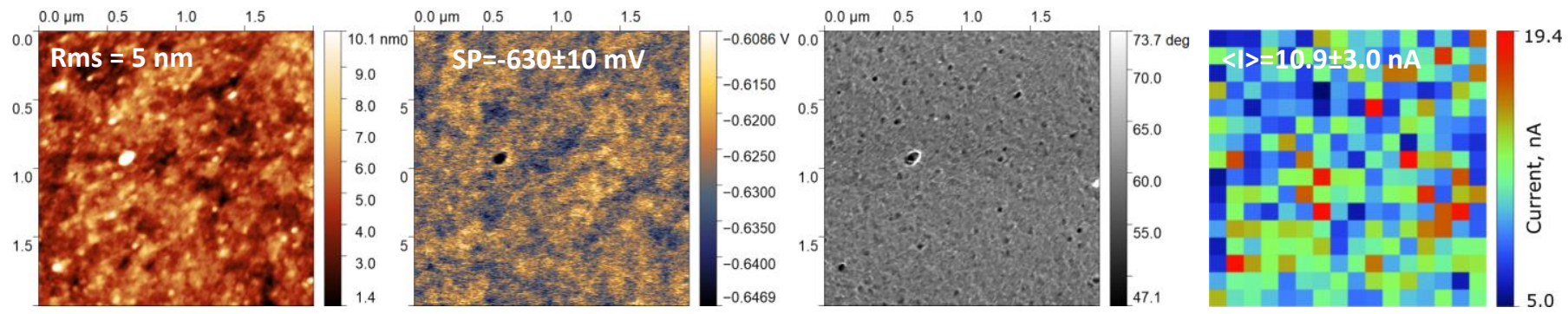

\section{Exposed films}
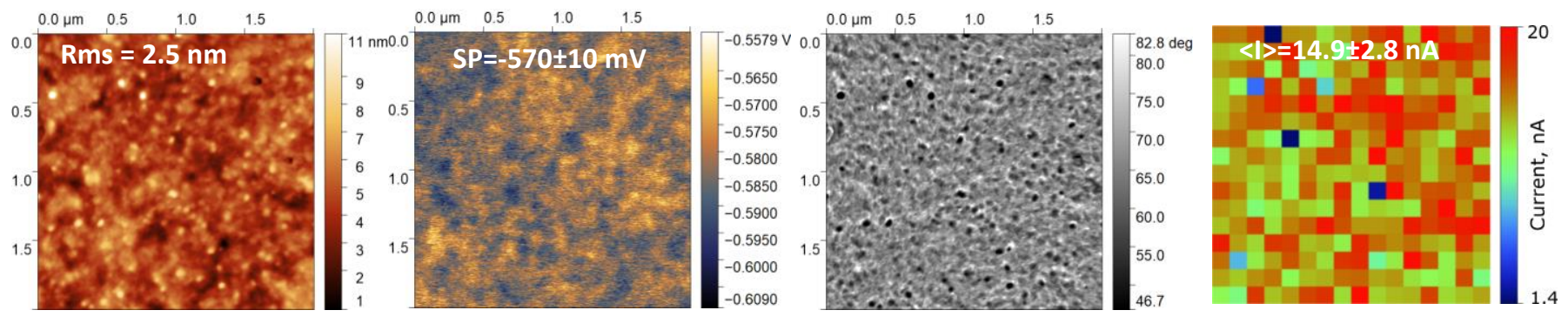

Figure S3. Left to right: AFM topography, surface potential, phase and current mapping images of $\mathbf{P} 1$ films before (top) and after (bottom) exposure to gamma rays (6500 Gy).

\section{Pristine films}
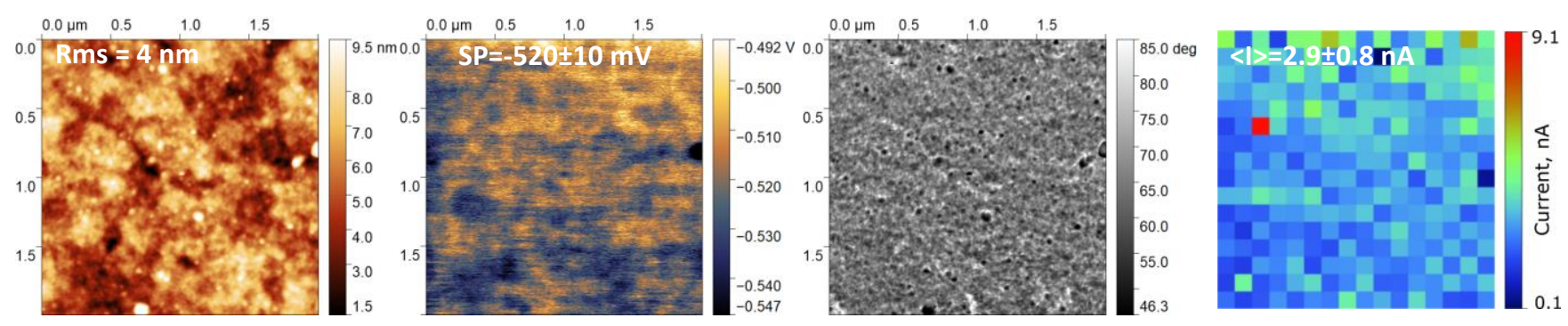

\section{Exposed films}
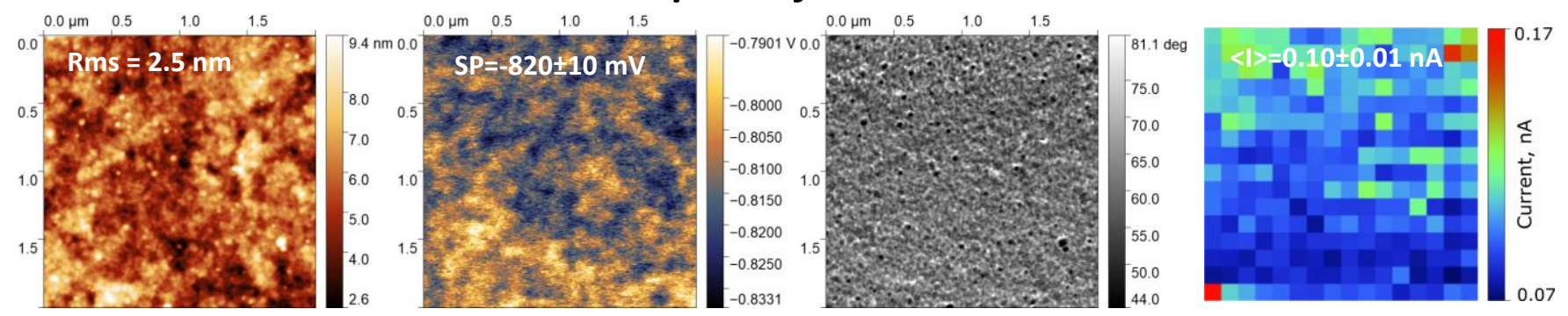

Figure S4. Left to right: AFM topography, surface potential, phase and current mapping images of $\mathbf{P 2}$ films before (top) and after (bottom) exposure to gamma rays (500 Gy). 


\section{Pristine films}

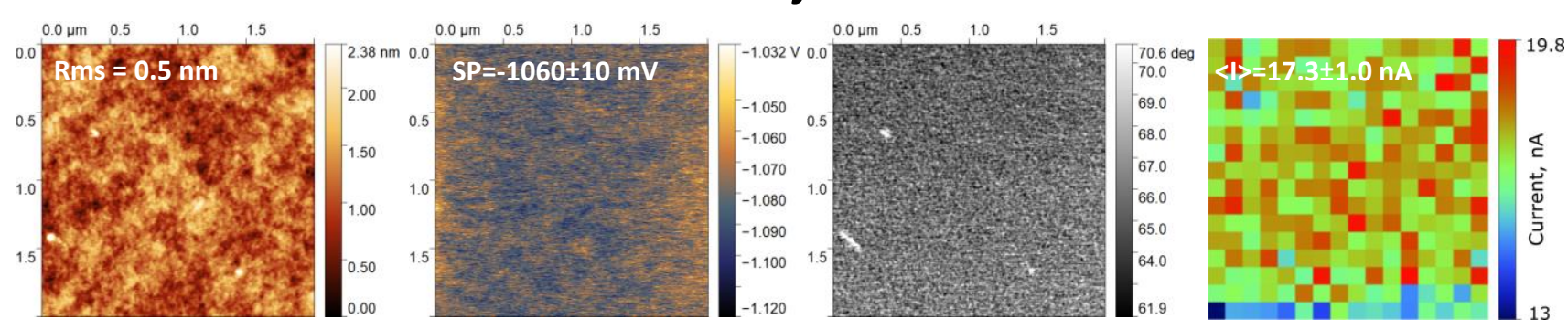

Exposed films

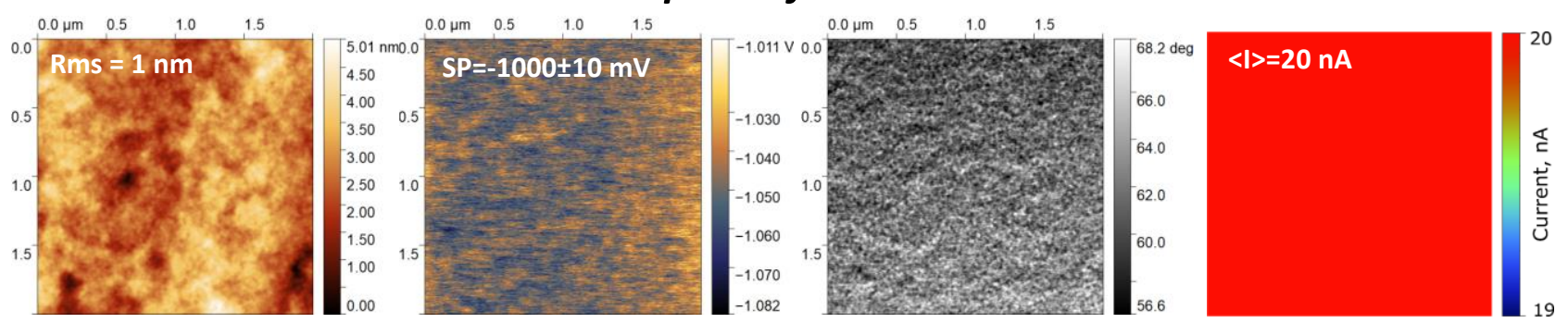

Figure S5. Left to right: AFM topography, surface potential, phase and current mapping images of [60]PCBM films before (top) and after (bottom) exposure to gamma rays (6500 Gy).
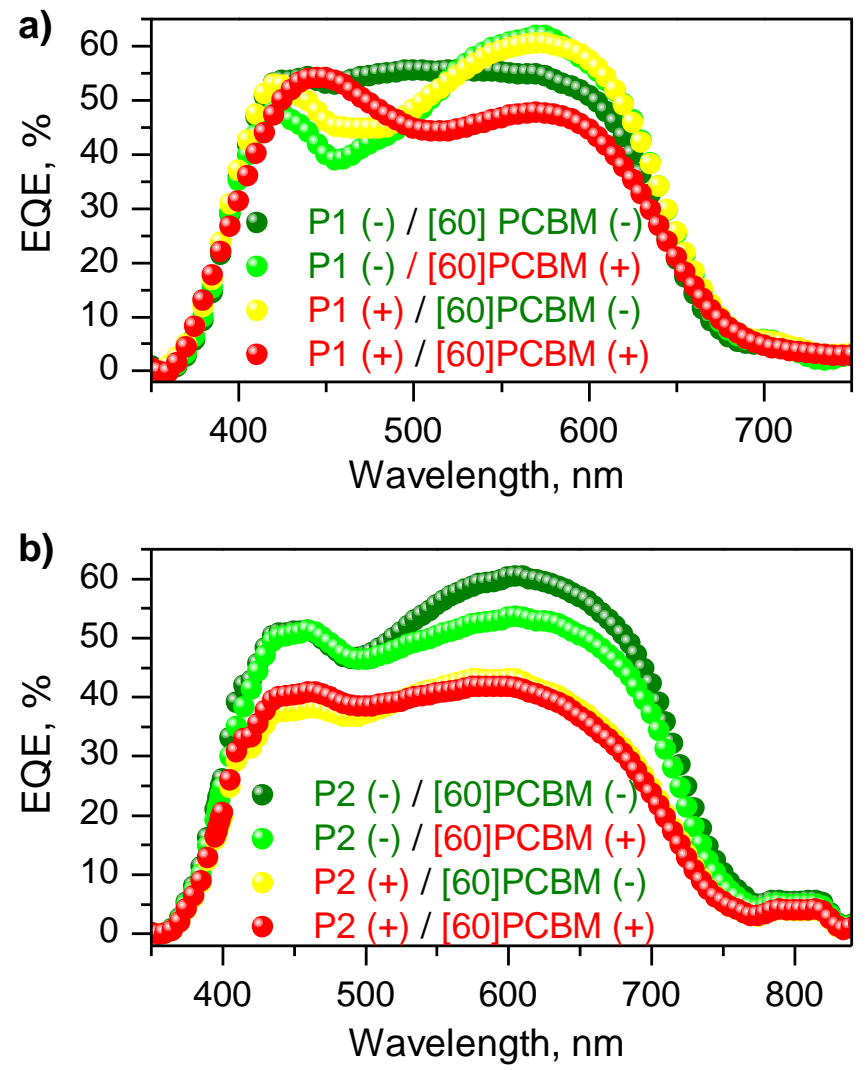

Figure S6. EQE spectra of organic solar cells based on the blends of non-exposed (-) and exposed to 200Gy (+) materials: P1/[60]PCBM (a) and P2/[60]PCBM (b). 

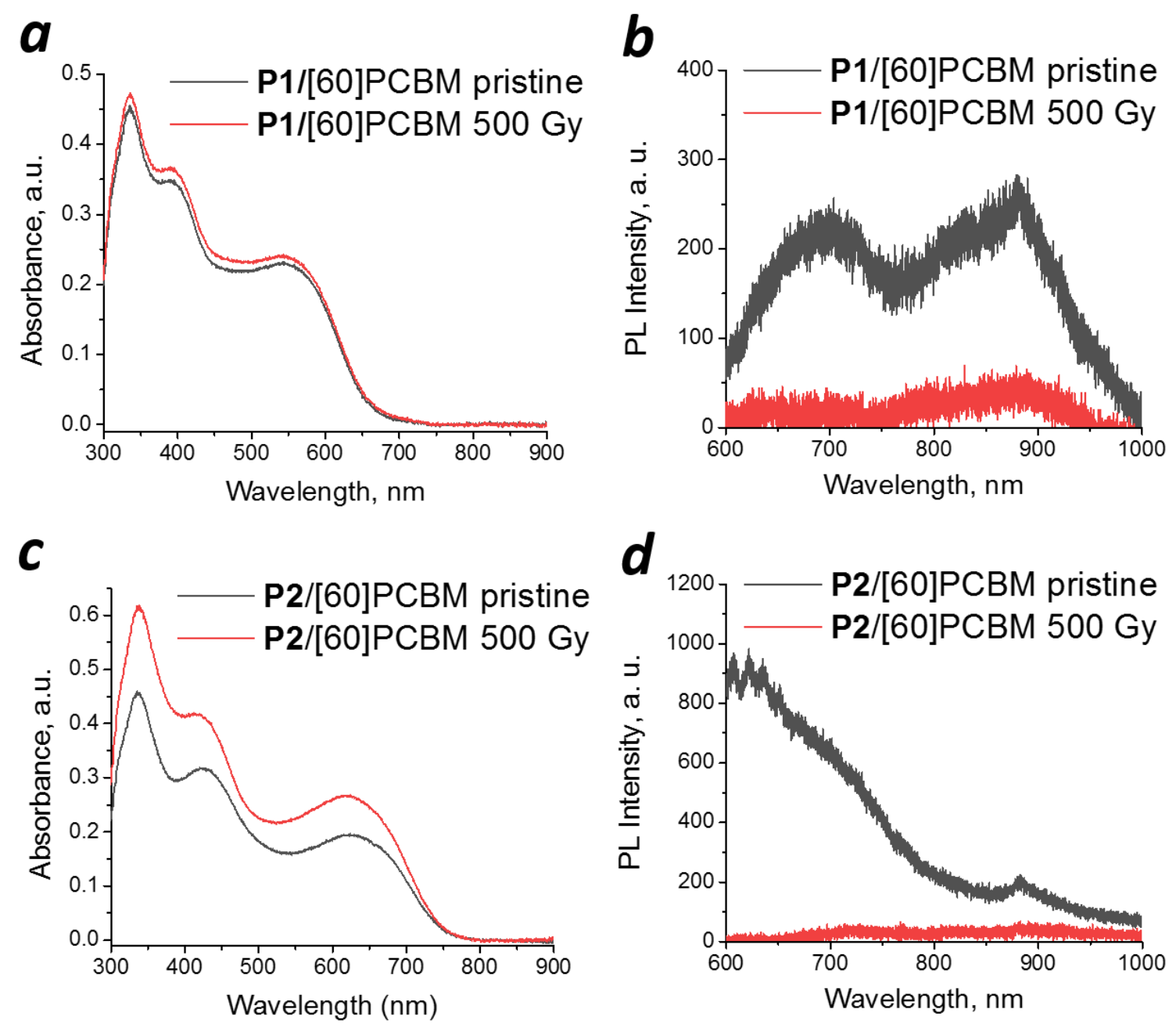

Figure S7 Evolution of the absorption $(a, c)$ and PL $(b, d)$ spectra of the P1/[60]PCBM (a-b) and P2/[60]PCBM (c-d) blend films 


\section{Pristine films}
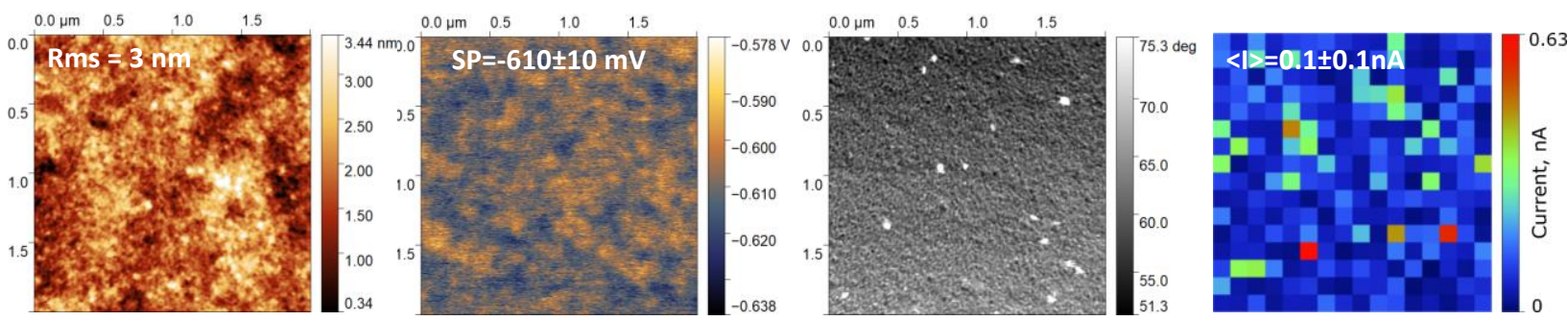

\section{Exposed films}

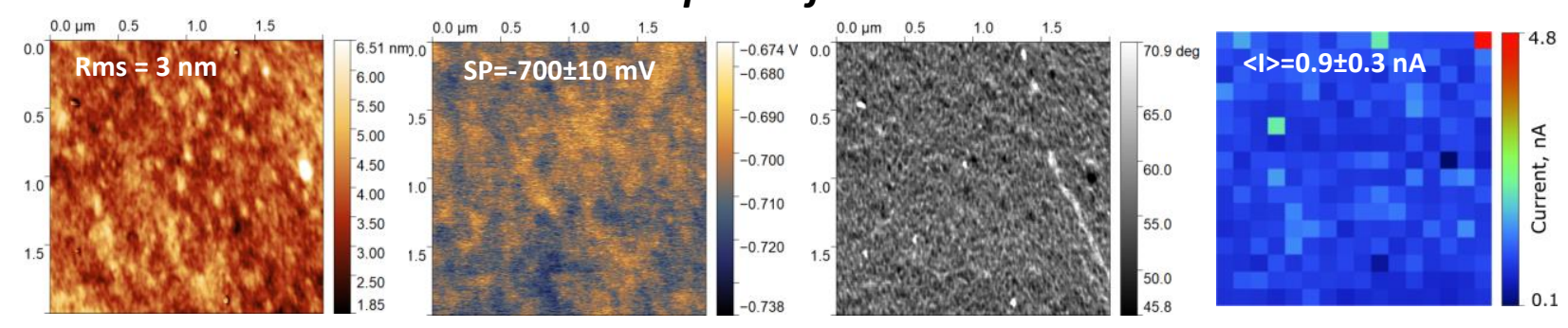

Figure S8. Left to right: AFM topography, surface potential, phase and current mapping images of P1/[60]PCBM films before (top) and after (bottom) exposure to gamma rays (6500 Gy)

\section{Pristine films}

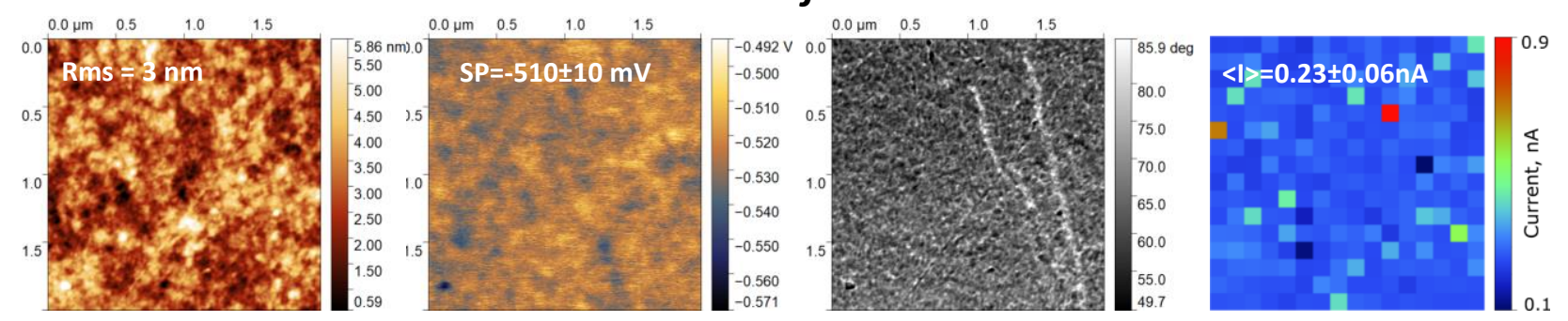

\section{Exposed films}

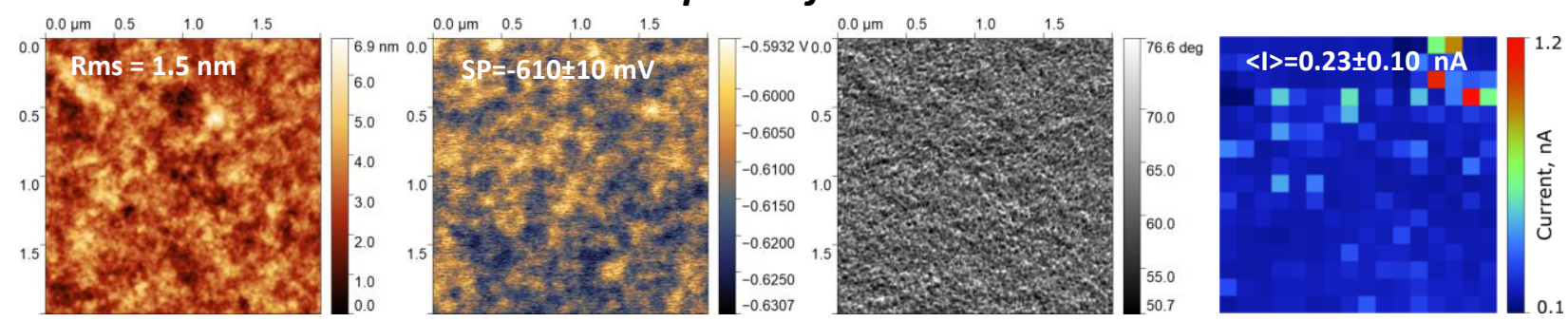

Figure S9. Left to right: AFM topography, surface potential, phase and current mapping images of P2/[60]PCBM films before (top) and after (bottom) exposure to gamma rays ( $500 \mathrm{~Gy}$ ) 


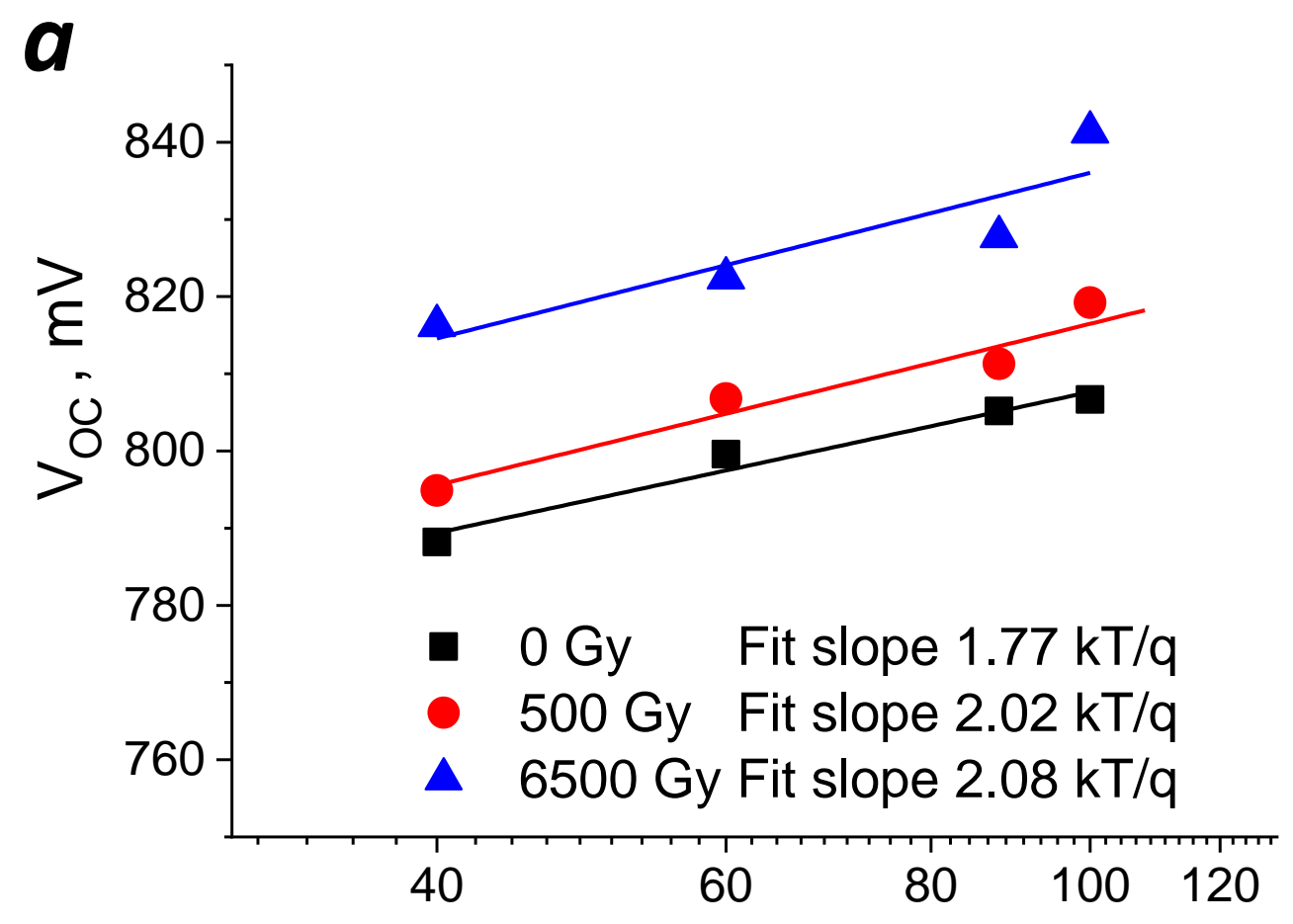

Light intensity, $\mathrm{mW} / \mathrm{cm}^{2}$

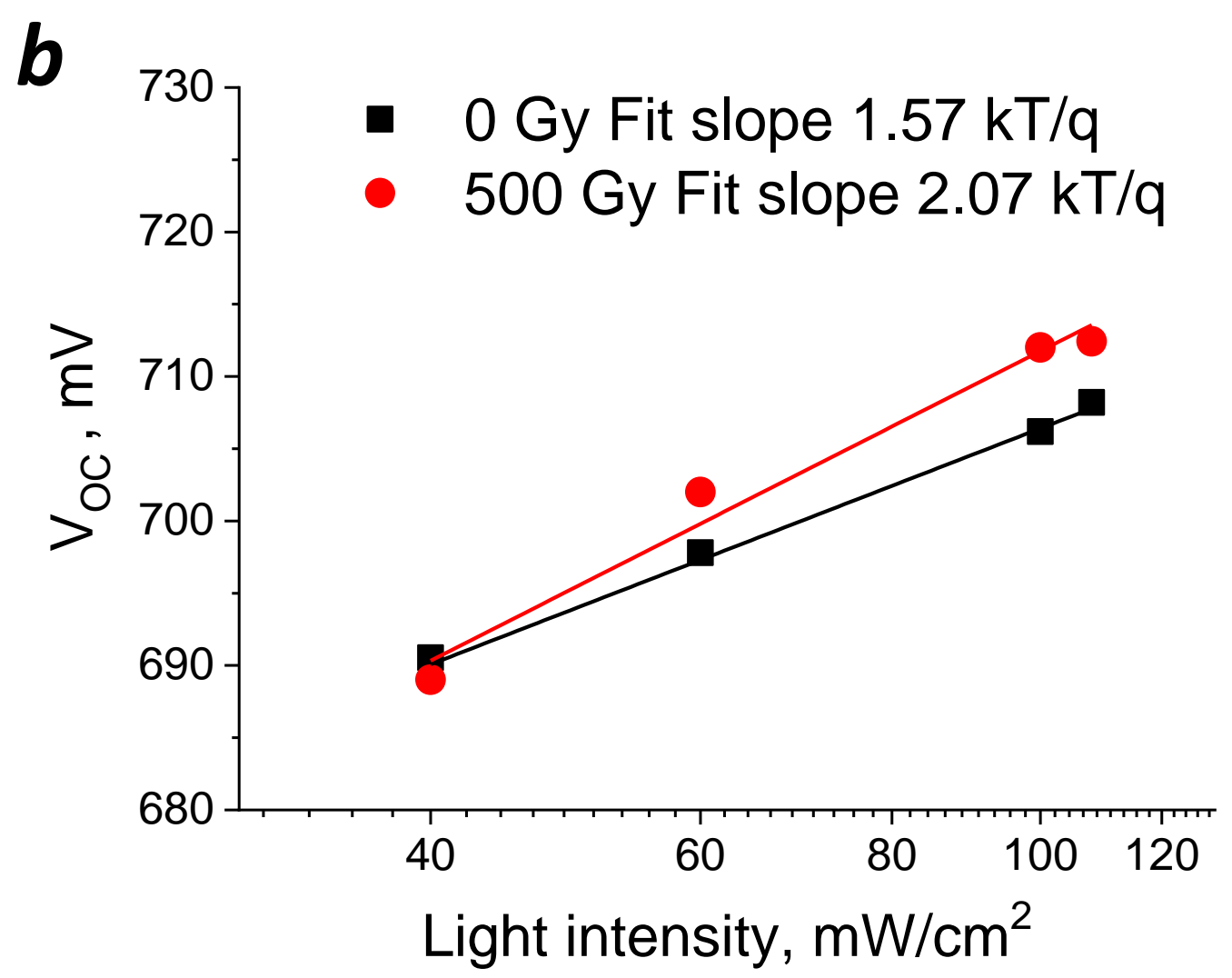

Figure S10. Voc vs. light intensity dependences for organic solar cells based on the pristine and exposed to gamma rays $\mathbf{P 1 / [ 6 0 ] P C B M ~ ( a ) ~ a n d ~ P 2 / [ 6 0 ] P C B M ~ ( b ) ~ b l e n d ~ f i l m s ~}$ 

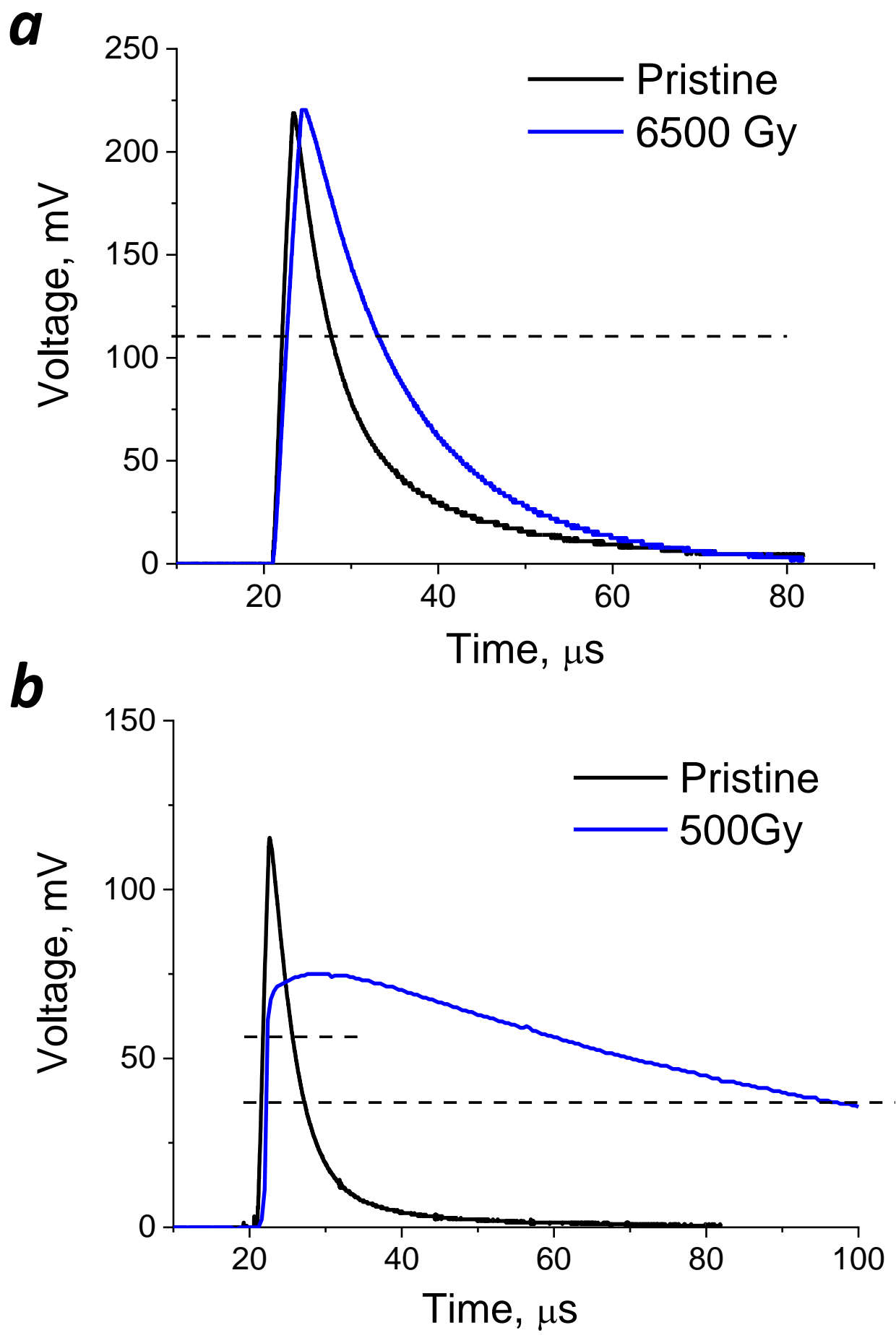

Figure S11. Transient photovoltage (TPV) profiles for organic solar cells based on the pristine and exposed to gamma rays P1/[60]PCBM (a) and P2/[60]PCBM (b) blend films 

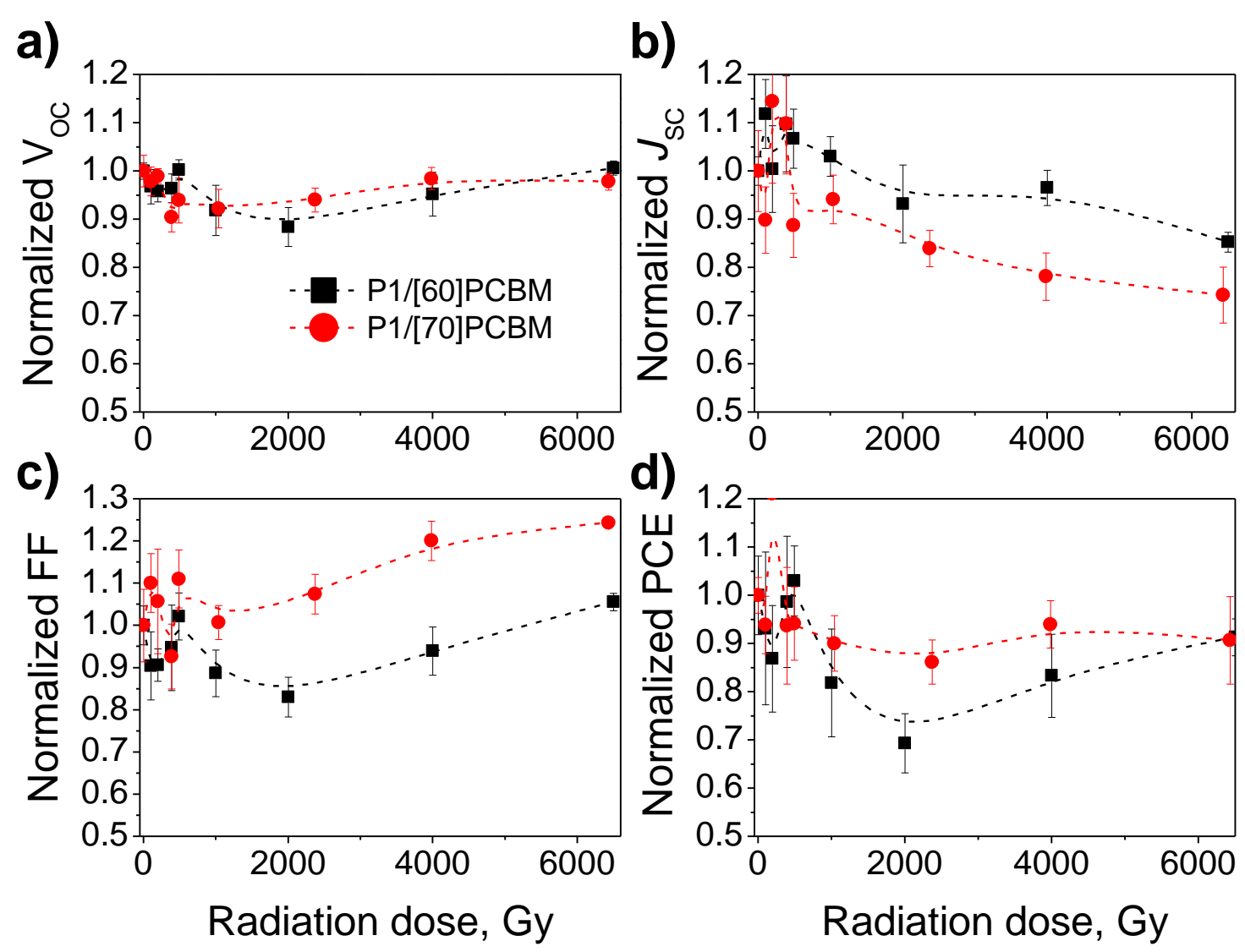

Figure S12. Normalized characteristics of the solar cells based on P1/[60]PCBM (black) and P1/[70]PCBM (red) plotted as functions of the radiation dose: open circuit voltage $V_{O C}(a)$, short circuit current density $J_{S C}(b)$, fill factor FF (c) and power conversion efficiency PCE (d) 
Table S1. Characteristics of organic solar cells before and after exposure of the samples to different doses of gamma rays

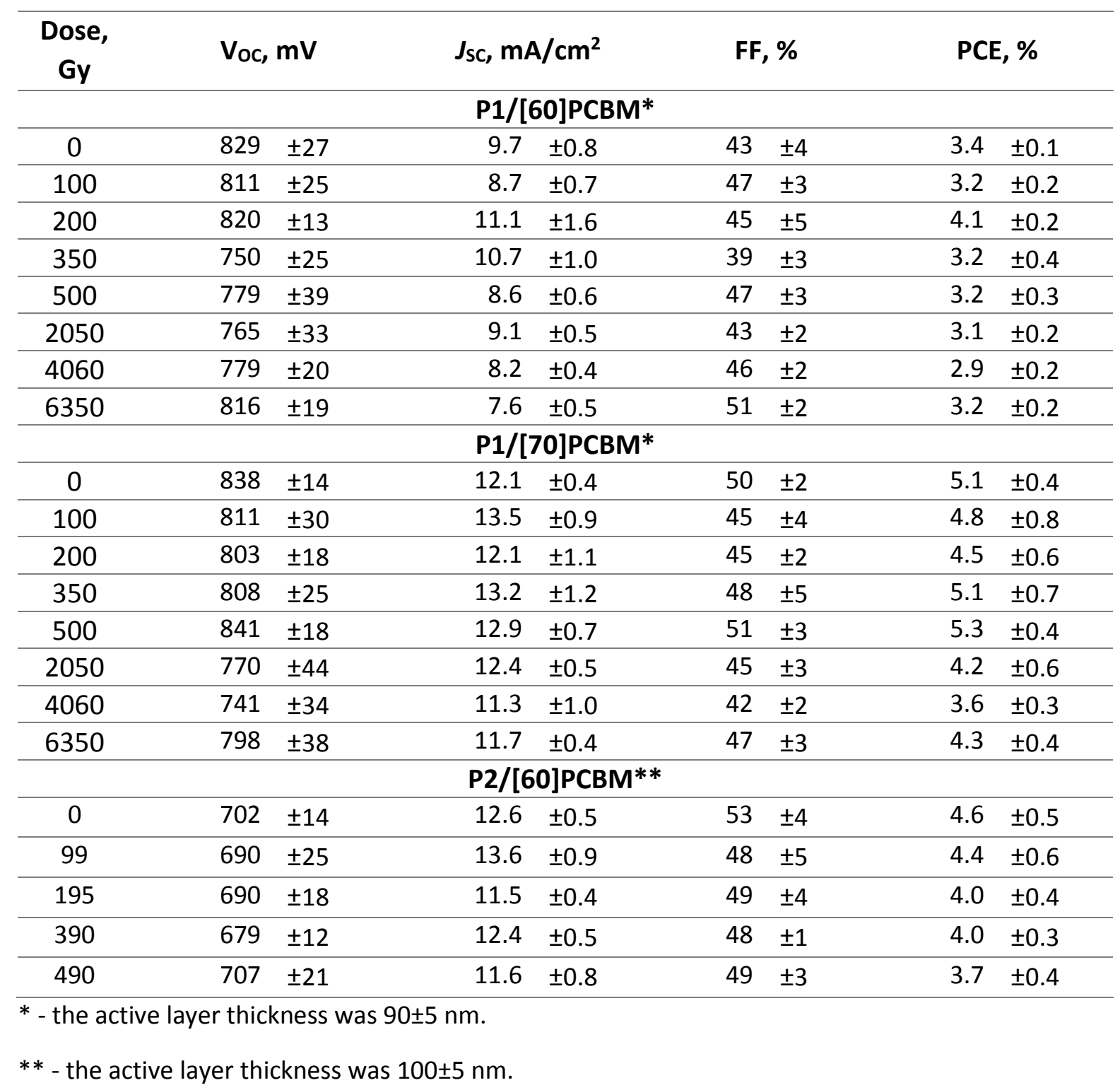

